\title{
BMJ Open Barriers and facilitators to patient and public engagement and recruitment to digital health interventions: protocol of a systematic review of qualitative studies
}

\author{
Siobhan O'Connor, ${ }^{1,2}$ Peter Hanlon, ${ }^{1}$ Catherine A O'Donnell, ${ }^{1}$ Sonia Garcia, ${ }^{3}$ \\ Julie Glanville, ${ }^{3}$ Frances S Mair ${ }^{1}$
}

To cite: O'Connor S,

Hanlon P, O'Donnell CA, et al. Barriers and facilitators to patient and public engagement and recruitment to digital health interventions: protocol of a systematic review of qualitative studies. BMJ Open 2016;6:e010895. doi:10.1136/bmjopen-2015010895

- Prepublication history for this paper is available online. To view these files please visit the journal online (http://dx.doi.org/10.1136/ bmjopen-2015-010895).

Received 22 December 2015 Revised 2 June 2016 Accepted 14 June 2016

CrossMark

For numbered affiliations see end of article.

Correspondence to Dr Frances S Mair; frances.mair@glasgow.ac.uk

\section{ABSTRACT}

Introduction: Patients and the public are beginning to use digital health tools to assist in managing chronic illness, support independent living and self-care, and remain connected to health and care providers. However, engaging with and enrolling in digital health interventions, such as telehealth systems, mobile health applications, patient portals and personal health records, in order to use them varies considerably. Many factors affect people's ability to engage with and sign up to digital health platforms.

Objectives: The primary aim is to identify the barriers and facilitators patients and the public experience to engagement and recruitment to digital health interventions. The secondary aim is to identify engagement and enrolment strategies, leading if possible to a taxonomy of such approaches, and a conceptual framework of digital health engagement and recruitment processes.

Methods: A systematic review of qualitative studies will be conducted by searching six databases: MEDLINE, CINAHL, PubMed, EMBASE, Scopus and the ACM Digital Library for papers published between 2000 and 2015. Titles and abstracts along with full-text papers will be screened by two independent reviewers against predetermined inclusion and exclusion criteria. A data extraction form will be used to provide details of the included studies. Quality assessment will be conducted using the Consolidated Criteria for Reporting Qualitative Research checklist. Any disagreements will be resolved through discussion with an independent third reviewer. Analysis will be guided by framework synthesis and informed by normalization process theory and burden of treatment theory, to aid conceptualisation of digital health engagement and recruitment processes.

Discussion: This systematic review of qualitative studies will explore factors affecting engagement and enrolment in digital health interventions. It will advance our understanding of readiness for digital health by examining the complex factors that affect

\section{Strengths and limitations of this study}

- This will be the first synthesis of qualitative studies to explore patients' and the public's experiences of engagement and recruitment to a broad range of digital health interventions.

- We will systematically identify and critically appraise the available evidence on this important topic, identify research findings and highlight any knowledge gaps.

- It is envisaged that the results of this review will contribute to a catalogue of barriers and facilitators that affect people's ability to engage with and sign up to digital health interventions; a taxonomy of engagement and enrolment strategies used if possible; and a preliminary conceptual model of digital health engagement and recruitment processes.

- This work will advance our understanding of the readiness of patients and the public for digital health.

- Findings may be limited by (1) the inclusion of English language publications as this could exclude potentially useful studies, which may result in cultural and publication bias, (2) the synthesis of qualitative studies which may result in the loss of some explanatory context that could limit the generalisability of findings or (3) the data analysis and synthesis which will be based on a sample of data extracted by the review team and not the original data.

patients' and the public's ability to take part. Trial registration number: CRD42015029846.

\section{INTRODUCTION}

Changing lifestyle patterns over the last century have seen growing numbers of 
people with one or more chronic illnesses, including heart disease, diabetes and cancer. Such conditions are the leading cause of death, disease and disability worldwide. $^{1-3}$ This combined with ageing populations, who have complex health and social care needs, is creating a huge resource burden on health systems ${ }^{4} 5$ with increases in healthcare usage and unplanned admissions. ${ }^{6}$ To become more sustainable, health services are beginning to move from treating illness in acute hospital settings to promoting more preventative care and the self-management of long-term conditions in the community where possible. ${ }^{7}$

Person-centred digital health interventions are being developed and trialled to further this agenda, by promoting active and healthy ageing, supporting individuals to manage long-term conditions at home and assisting them to remain connected with health and care providers. Examples include telehealth and telecare systems, ${ }^{9}$ electronic personal health records (PHRs) ${ }^{10}$ and mobile health applications or 'apps'11 among others. It is hoped that these types of digital products and services could lead to better health outcomes and to a reduction in the usage and cost of primary, secondary and tertiary health services. $^{12}$

Many of these digital health interventions have only been evaluated in small pilot studies or randomised controlled trials (RCTs). While the results of these can sometimes demonstrate positive outcomes, ${ }^{13}$ many people choose not to enrol as they can experience numerous barriers, some of which relate to the complex recruitment procedures in trials. ${ }^{14-16}$ While nonparticipation is a significant issue, ${ }^{17} 18$ there are also many factors that can facilitate patients and the public to engage with and sign up to digital health platforms. ${ }^{19} 20$ Although there is a large literature on difficulties recruiting to trials, ${ }^{21}$ many challenges only emerge when technologies are scaled up and implemented in 'real-world' complex health systems. ${ }^{22}{ }^{23}$ This is an important distinction, as RCTs have predefined protocols and strict inclusion criteria that can often mask wider implementation issues. ${ }^{24}{ }^{25}$ Problems that can arise when technologies are scaled up begin in the initial phases of implementing a digital health intervention in practice, when engaging with different stakeholders, when encouraging people to register for digital products and services on offer (or which might be offered in the future) and then facilitating them to sign up for it. The journey by which such technologies are implemented can span a long timeline of individual and organisational change processes, not all of which occur sequentially. ${ }^{26}$ As a result, this review is focusing on the initial phases of rolling out digital health products and services, in particular the engagement and recruitment of users. By engagement, we mean the processes by which patients and the public become aware of and understand digital health interventions, for example, through promotional efforts and marketing campaigns. Recruitment, on the other hand, encompasses the processes that people are involved in when enrolling or signing up to digital health products or services, such as actively filling out paper-based registration forms or creating online profiles or accounts. There has as yet been no attempt to synthesise what this literature tells us about the key challenges or the outstanding research gaps surrounding patient and public engagement and recruitment to digital health.

There have been repeated calls for more research that synthesises the findings from qualitative evidence to support policy and the translation of research into clinical practice. ${ }^{27}$ For example, a synthesis of qualitative research can aid in the understanding of complex interventions as well as human experiences and behaviour, all of which can be used to inform health policy and practice. $^{28} 29$ The volume of qualitative systematic reviews has been growing slowly over the last number of years $^{30}$ due in part to the work of the Cochrane Qualitative and Implementation Methods Groups (CQIMG) and others who are championing the role qualitative synthesis can play in building the base for effective evidence that can be adopted and implemented in everyday practice. ${ }^{31} 32$ At present, there are limited, if any published, systematic reviews of qualitative studies looking at different aspects of person-centred digital health interventions. In particular, there is no systematic review on the topic of digital health engagement and recruitment, although it has been highlighted as a research gap that needs attention. ${ }^{14}{ }^{25}$ An understanding of the barriers patients and the public experience when trying to engage and enrol in digital health products and services, would help ensure the full value of these interventions can be realised. Similarly, robust evidence on the factors that facilitate this process could improve recruitment to future digital health initiatives.

In addition, theoretical frameworks have been used previously to aid many types of qualitative synthesis ${ }^{24} 3334$ as established models can help to explain the phenomenon under consideration and make the findings of the synthesis more accessible to application in practice. ${ }^{35} 36$ Therefore, this review will incorporate two empirically grounded sociological theories, normalization process theory (NPT) and burden of treatment theory (BOTT), as they are relevant to understanding how individuals embed new interventions in everyday routine and the burden that this process entails. ${ }^{37} 38$ NPT in particular has been widely used in eHealth implementation research to examine different stages of the process through its four main constructs: 'coherence', 'cognitive participation', 'collective action' and 'reflexive monitoring' (see figure 1). ${ }^{39}{ }^{40}$ BOTT was built on the conceptual foundations of NPT to expand our knowledge of the difficulties patients experience when coping with new treatment modalities and enacting self-care strategies ${ }^{41}$ so they are both directly applicable to this qualitative synthesis. This systematic review of qualitative studies aims to identify and synthesise currently available knowledge about barriers and facilitators to engagement and recruitment across a range of digital health interventions 


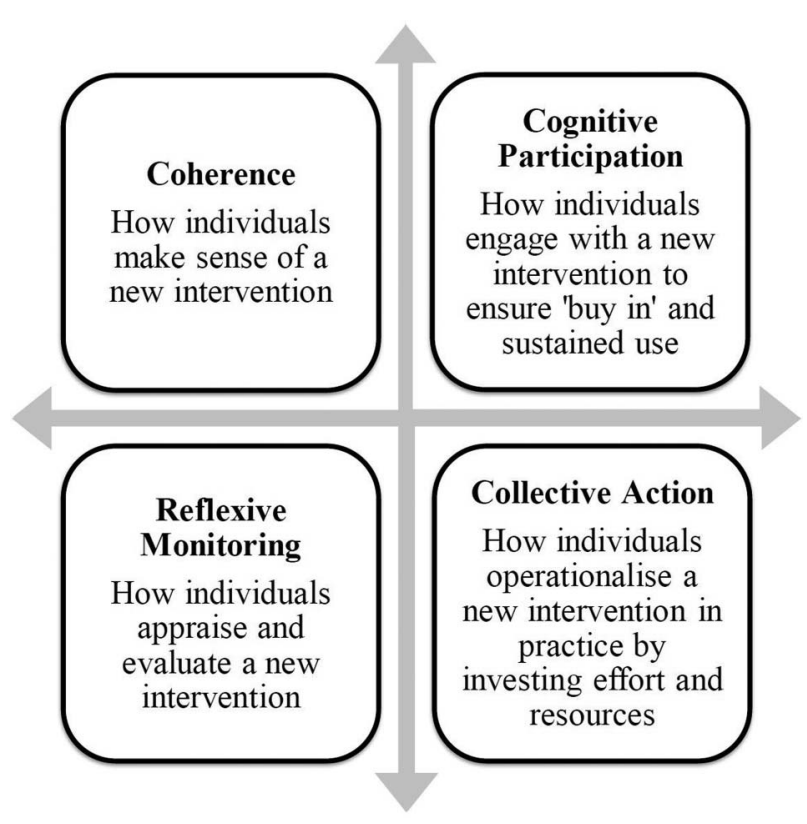

Figure 1 Four constructs of normalisation process theory (NPT).

and highlights knowledge gaps and areas for further research. If possible, we will also aim to develop a taxonomy of digital health engagement and recruitment strategies used and a preliminary conceptual model of digital health engagement processes. Arguably, a better understanding and detailing of these difficulties and the processes involved will help researchers and those in industry design better systems and enrolment strategies and inform health service managers and policymakers of changes that need to be made to improve digital health engagement and recruitment.

\section{Objectives}

Primary

To examine the factors (barriers and facilitators) that affect patients' and the public's ability to engage with and enrol in digital health interventions.

\section{Secondary}

To determine what engagement and recruitment strategies have been used to sign people up to digital health products and services.

\section{METHODS}

This protocol was written in accordance with the Preferred Reporting Items for Systematic Reviews and Meta-analysis Protocols (PRISMA-P) checklist. $^{42}$ Reporting of the full systematic review will follow the Enhancing Transparency in Reporting the Synthesis of Qualitative Research (ENTREQ) statement. ${ }^{43}$ An interdisciplinary team of researchers will be involved in the systematic review with expertise ranging from general practice and primary care, to nursing, health informatics and information science. Members of this team have conducted and published numerous highquality systematic reviews and the collective skill set will enable a robust review to be carried out. ${ }^{33}$ An initial 'scoping search' will be undertaken to help identify key papers and search terms that are relevant to the research topic. This process will be carried out by conducting a preliminary search of online bibliographical databases via Ovid; the use of the 'related articles' function in PubMed, reference and footnote tracking of relevant papers found, citation tracking of relevant papers found, the use of personal knowledge, and consultation with experts in the field. The results will help to inform the initial design of the search strategy for the review which will be piloted and refined as appropriate. Figure 2 outlines the detailed workflow of the proposed review.

\section{Search strategy}

A team of information specialists at the York Health Economics Consortium (YHEC), who specialise in conducting systematic reviews, will be consulted to assist with the design of the search strategy. There is likely to be three groups of search terms, referring to digital health interventions, engagement and recruitment, and factors that affect these processes, that is, barriers and facilitators (table 1). Guidance will be sought from the Cochrane Handbook for Reviews of Interventions on the most robust way to systematically search the literature, $^{44}$ and other novel strategies such as text mining ${ }^{4546}$ will be explored and applied where appropriate. The following six electronic databases, CINAHL, (EBSCHOHost), PubMed, Medline, EMBASE, Scopus and the ACM Digital Library, will be systematically searched to identify published peer-reviewed scientific literature that are relevant to the research objectives. YHEC will run searches, remove duplicate citations and provide an EndNote database file of citations for screening.

The challenges of searching the qualitative literature have been well documented ${ }^{47-49}$ and studies have shown that traditional database searching can reveal as little as $30 \%$ of research papers that are relevant to the topic under review. ${ }^{50}$ Therefore, other search methods will be used to supplement the results of the systematic review and identify relevant studies. These will include reference or footnote tracking; using the 'related articles' function in PubMed; citation tracking; personal knowledge and personal contacts and contacting experts in the field.

\section{Eligibility criteria}

The review will adhere to the following criteria. These were developed using a modified population, intervention, control and outcome (PICO) framework (see table 2).

Papers will be included if they meet the PICO criteria. Below is a detailed description of the inclusion and exclusion criteria. 


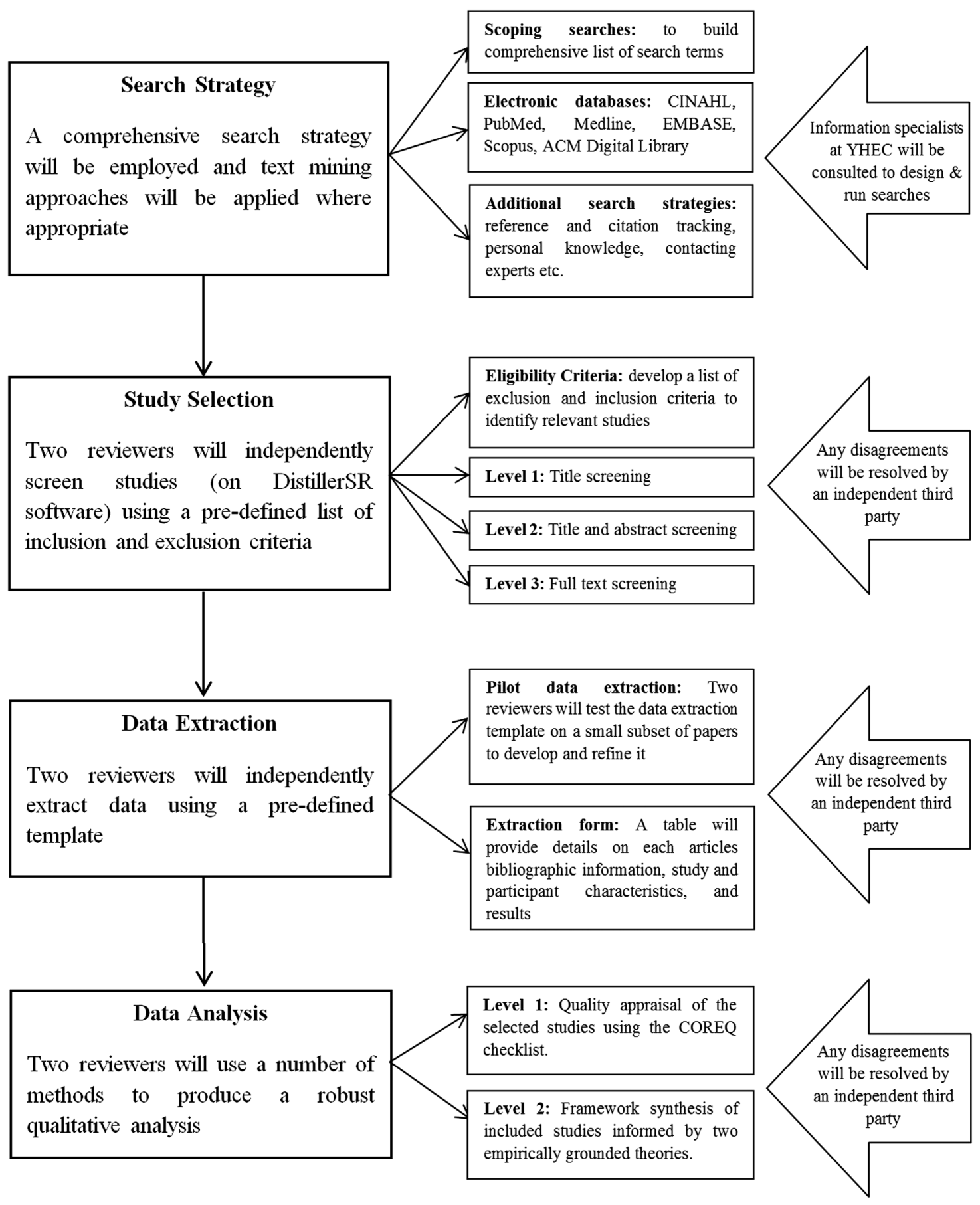

Figure 2 Proposed workflow of the qualitative systematic review. COREQ, Consolidated Criteria for Reporting Qualitative Research; YHEC, York Health Economics Consortium.

1. Types of studies: Original qualitative studies, studies involving secondary analysis of qualitative data or qualitative studies as part of a mixed methods study (eg, a major component must be qualitative and describe a qualitative methodology). Certain types of studies will be excluded from the review including those using the following methodologies:

A. literature or systematic reviews and meta-analyses;

B. descriptive case studies; lexical studies that analyse natural language data presented as qualitative results;
C. qualitative studies using questionnaires or other methods that do not involve direct contact or observation of participants;

D. commentary articles written to convey opinion or stimulate research/discussion with no research component;

E. studies describing an individual's experience(s) in an RCT. This review is focusing on barriers and facilitators to engagement and enrolment to digital health interventions rather than the wider 


\begin{tabular}{ll} 
Table 1 & Search strategy \\
Search & $\begin{array}{l}\text { Search terms that will be modified for use } \\
\text { across multiple databases }\end{array}$ \\
\hline \#1 & Search Electronic Health Records [MeSH Terms] \\
$\# 2$ & Search Cellular Phone [MeSH Terms] \\
$\# 3$ & Search Social Networking [MeSH Terms] \\
$\# 4$ & Search Telemedicine [MeSH Terms] \\
$\# 5$ & \#1 OR \#2 OR \#3 OR \#4 \\
$\# 6$ & Search recruit* \\
$\# 7$ & Search enrol* \\
$\# 8$ & Search participat* \\
$\# 9$ & Search engage* \\
$\# 10$ & \#6 OR \#7 OR \#8 OR \#9 \\
$\# 11$ & Search barrier* \\
$\# 12$ & Search challenge* \\
$\# 13$ & Search impediment* \\
$\# 14$ & Search facilit* \\
$\# 15$ & \#11 OR \#12 OR \#13 OR \#14 \\
$\# 16$ & \#5 AND \#10 AND \#15 \\
\hline
\end{tabular}

literature on recruitment to clinical trials that has been addressed in previous reviews. ${ }^{21}$

2. Types of eHealth interventions: Any health intervention delivered by a digital technology (hypothetical or in development, simulated or real world), which takes information from patients or the public, or provides some form of advice or feedback about their health. This includes, but is not limited to, web-based interventions on personal computers (PCs) or mobile platforms, mobile health applications or apps, patient portals, PHRs and interventions delivered by short message service (SMS) or interactive voice recognition (IVR). Certain technologies will be excluded from the review including those whose primary intervention is telephone based with no additional technological function (eg, telephone counselling or triaging service), internet based with no additional interactive function (eg, searching for health information online), or an implantable device that is remotely monitored.

3. Types of participant: Any individual (adult or child). This includes patients, the public and health professionals who would be aware of the experiences of these stakeholder groups.

4. Types of settings: Any 'usual' setting (hypothetical or in development, simulated or real world), such as primary, secondary or tertiary care, home or workplace.

5. Phase of implementation: Qualitative research which explores the initial phases of implementation, that is, engagement and recruitment phase, before individuals start using a digital health intervention. This can span from gauging an individual's readiness for a digital health intervention, to the initial marketing or reach of the initiative, to actively signing people up to use the technology so they are registered on the digital application or system. Therefore, we will not explore: pre-engagement work based solely around
Table 2 PICO criteria for including studies

Population

Any individual (adult or child). This includes patients, the public and health professionals who would be aware of the experiences of these stakeholder groups.

Intervention Any health intervention delivered by a digital technology (hypothetical or in development, simulated or real world) which takes information from people or provides some form of advice or feedback about their health. This includes, but is not limited to:

- web-based interventions on PCs or mobile platforms,

- mobile health applications or apps,

- patient portals or personal health records,

- interventions delivered by SMS or IVR.

Control None.

Outcome Qualitative data on the factors (barriers and facilitators) to engagement and recruitment. Qualitative data on engagement and enrolment strategies.

Study type Original qualitative studies, studies involving secondary analysis of qualitative data or qualitative studies that are part of a mixed-methods study. The study must have direct contact with individuals or direct observation using any form of qualitative method.

Setting Any 'usual' setting (hypothetical or in development, simulated or real world) such as primary, secondary or tertiary care, the home or workplace.

Timing or phase of Engagement or recruitment implementation phase only.

IVR, interactive voice recognition; PC, personal computer; PICO, population, intervention, control and outcome; SMS, short message service.

designing the interface and functionality of a digital health intervention; patients' or the public's use of these types of technologies; why they drop out or fail to continue using them (non-usage or attrition) $)^{52}$ or sustain their use of them (retention) $;^{53}$ their attitudes or beliefs towards a digital health intervention or their satisfaction with it, except as pertaining directly to engagement or recruitment.

6. Date of publication: Between 1 January 2000 and 19 August 2015.

7. Language: English. 


\section{Screening}

Each title and abstract will be screened by two independent reviewers using DistillerSR software (DistillerSR. Systematic Review and Literature Review Software from Evidence Partners. 2016. https://distillercer.com/products/distillersr-systematic-review-software/). All journal articles that meet the inclusion criteria set out above will be obtained in full-text format for further screening and assessment. Two independent reviewers will conduct the second level full paper screening by applying the inclusion and exclusion criteria. A third party will be involved to resolve any conflicts that occur in determining the relevance of the titles, abstracts and full-text papers, so that a consensus over inclusion and exclusion of each article can be reached. A flow diagram will be used to report the selection process and reasons for exclusion as suggested by the PRISMA guidelines. ${ }^{54}$

\section{Data extraction}

Data extraction will be carried out using a comprehensive, standardised extraction template that will be designed based on the specific characteristics of this review, including aims of the study, design and methodological approach taken and key findings such as barriers and facilitators and a description of engagement and recruitment strategies. It will be piloted on a subset of relevant papers and refined where appropriate. The extraction process will be conducted by two individual reviewers based on the relevant articles previously identified through the screening process. A third party will be involved where disagreements arise over the relevancy of the data to the review topic which will assist in reaching consensus and creating a robust dataset. This will result in a table that provides the following details:

- Bibliographic information such as the journal name, year, volume and page numbers;

- Study characteristics such as the type of technology and qualitative approach taken;

- Participant characteristics (sociodemographic) and information about the number and type of individuals who signed up or declined to take part;

- Main findings such as the barriers and facilitators to engagement or recruitment;

- Details of engagement or enrolment strategies used.

\section{Quality assessment of included studies}

The CQIMGs and others recommend critically appraising qualitative research as it helps assess whether the study adequately addresses the different dimensions of research quality such as credibility, transferability, dependability and confirmability ${ }^{55}{ }^{56}$ Although some are sceptical of this approach, ${ }^{57}$ a range of tools and checklists have been devised for this purpose. One such checklist is the Consolidated Criteria for Reporting Qualitative Research (COREQ), which offers a list of questions for assessing qualitative studies. ${ }^{58}{ }^{59}$ While the review team acknowledges that the assessment of qualitative research involves well-honed interpretative skills rather than relying solely on simplistic scoring criteria, ${ }^{60}$ the COREQ checklist will be applied to this review as it can enable a rapid evaluation of different types of qualitative studies and their major strengths or weaknesses. Two reviewers will independently assess the quality of the relevant studies and discussion will be used to resolve any conflicts. An independent third reviewer will be contacted if necessary to settle unresolved disagreements. No study will be excluded based on quality assessment as methodologically weak studies may still offer valuable insights. ${ }^{61}$

\section{Data analysis/synthesis}

This review will synthesise qualitative literature on patients' or the public's experiences of digital health engagement and recruitment. Our analyses will be informed by framework synthesis as it allows a priori model to be used to facilitate analysis. ${ }^{62}$ As outlined, NPT $^{37} 3940$ will be used during coding and synthesis, due to its highly conceptual relevance to the review topic. This process will be guided by the framework approach, which follows a five-stage process: (1) familiarisation, (2) identifying a thematic framework, (3) indexing, (4) charting and (5) mapping and interpretation. ${ }^{63}$ This series of analytical steps will facilitate in-depth interpretation of data until a rich and coherent understanding emerges. Overarching concepts will be mapped onto constructs from NPT and findings viewed through the lens of BOTT, although we will be open to the identification and coding of emergent themes that sit outside these theoretical frameworks, in order to inform development of a preliminary model of digital health engagement and recruitment. ${ }^{64}$ The engagement and enrolment strategies identified during the analyses will also be classified, if possible, to create a taxonomy of approaches. These might include traditional forms of mass media and recruitment via health professionals, to more contemporary methods using social media and online advertising to reach and enrol large numbers of people. NVivo software will be used to aid analysis. ${ }^{65}$

\section{DISCUSSION}

Engaging and recruiting patients and the public to digital health interventions is a complex process that needs to be fully explored if we are to capitalise on the value these technologies can offer. To date, existing research on this topic has not been synthesised. The systematic review of qualitative studies aims to address this gap in the scientific literature by providing insights into what helps and hinders patients and the public to engage and enrol in digital health products and services. This will inform our understanding of the readiness of these important stakeholder groups for digital health. We will use the findings of our analysis and synthesis work to create a catalogue that describes the barriers and facilitators that affect people's ability to sign up to digital health interventions, and if possible, devise a 
taxonomy of digital health engagement and enrolment strategies. We will produce a preliminary conceptual model of digital health engagement and recruitment processes. We anticipate that this work will be highly relevant to a wide range of stakeholders including researchers and industry who are developing and evaluating person-centred digital health interventions; health professionals who may want to recommend new electronic systems and applications to people; patients and the public who want to engage with and sign up to use novel technologies; local and national health services who wish to implement new digital services and enrol a variety of users on them; and policymakers who wish to address barriers to digital health engagement and recruitment. The results of this systematic review will be widely disseminated through publication in peerreviewed, open-access academic journals, research meetings, conference presentations and social media. Public engagement is also important and will be achieved through knowledge translation events and activities such as seminars, workshops and the use of social media.

\section{Author affiliations \\ ${ }^{1}$ General Practice and Primary Care, Institute of Health and Wellbeing, University of Glasgow, Glasgow, UK \\ ${ }^{2}$ School of Nursing, Midwifery and Social Work, University of Manchester, Manchester, UK \\ ${ }^{3}$ York Health Economics Consortium, University of York, York, UK}

Twitter Follow Siobhan 0'Connor at @shivoconnor

Contributors SOC, FSM and CAOD conceptualised the study and designed the review. JG and SG designed the search strategy with input from SOC and FSM. SOC wrote the first draft of the protocol with input from FSM and CAOD. SOC, FSM, CAOD, PH, JG and SG contributed to the writing of the final manuscript. All authors read and approved the final version of the manuscript. FSM is the guarantor of this research.

Funding The authors would like to acknowledge Innovate UK (formerly known as the UK Technology Strategy Board) who partly funded this work through a research grant. The funder had no part in the design of this review and the views expressed in this paper are those of the authors and not necessarily those of Innovate UK.

Competing interests None declared.

Provenance and peer review Not commissioned; externally peer reviewed.

Data sharing statement All data collected that are not published as part of the qualitative systematic review will be kept on electronic management applications (EndNote, Excel, NVivo) on secure computer systems at the University of Glasgow. The data will be available to the research team and will be made available to other researchers on request.

Open Access This is an Open Access article distributed in accordance with the Creative Commons Attribution Non Commercial (CC BY-NC 4.0) license, which permits others to distribute, remix, adapt, build upon this work noncommercially, and license their derivative works on different terms, provided the original work is properly cited and the use is non-commercial. See: http:// creativecommons.org/licenses/by-nc/4.0/

\section{REFERENCES}

1. Yach D, Hawkes C, Gould CL, et al. The global Burden of chronic diseases: overcoming impediments to prevention and control. JAMA 2004;291:2616-22.

2. World Health Organisation (WHO). Preventing chronic disease a vital investment. Geneva: WHO, 2005.
3. Barnett K, Mercer SW, Norbury M, et al. Epidemiology of multimorbidity and implications for healthcare, research, and medica education: a cross-sectional study. Lancet 2012;380:37-43.

4. Beaglehole R, Bonita R, Horton R, et al. Priority actions for the non-communicable disease crisis. Lancet 2011;377:1438-47.

5. World Health Organisation (WHO). Prevention and control of noncommunicable diseases in the European Region: a progress report. Copenhagen: WHO Regional Office for Europe, 2013.

6. Payne RA, Abel GA, Guthrie B, et al. The effect of physical multimorbidity, mental health conditions and socioeconomic deprivation on unplanned admissions to hospital: a retrospective cohort study. CMAJ 2013;185:E221-8.

7. Bodenheimer $\mathrm{T}$, Lorig $\mathrm{K}$, Holman $\mathrm{H}$, et al. Patient self-management of chronic disease in primary care. JAMA 2002;288:2469-75.

8. National Health Service (NHS). NHS 2010-2015: from good to great preventative, people-centred, productive. Crown: London, 2009. (December 15 2015). https://www.gov.uk/government/publications/ nhs-2010-to-2015-from-good-to-greatpreventative-people-centredproductive

9. May C, Mort M, Williams T, et al. Health technology assessment in its local contexts: studies of telehealthcare. Soc Sci Med 2003;57:697-710

10. Pagliari C, Detmer D, Singleton P. Potential of electronic personal health records. BMJ 2007;335:330.

11. Free C, Phillips G, Galli L, et al. The effectiveness of mobile-health technology-based health behaviour change or disease management interventions for healthcare consumers: a systematic review. PLOS Med 2013;10:e1001362.

12. Hunkeler EM, Meresman JF, Hargreaves WA, et al. Efficacy of nurse telehealthcare and peer support in augmenting treatment of depression in primary care. Arch Fam Med 2000;9:700-8.

13. Steventon A, Bardsley M, Billings $\mathrm{J}$, et al. Effect of telehealth on use of secondary care and mortality: findings from the Whole System Demonstrator cluster randomised trial. BMJ 2012;344:e3874.

14. Sanders C, Rogers A, Bowen R, et al. Exploring barriers to participation and adoption of telehealth and telecare within the Whole System Demonstrator trial: a qualitative study. BMC Health Serv Res 2012;12:220.

15. Varsi C, Gammon D, Wibe T, et al. Patients' reported reasons for non-use of an internet based patient-provider communication service: qualitative interview study. J Med Internet Res 2013;15: e246.

16. Buis LR, Janney AW, Hess ML, et al. Barriers encountered during enrollment in an internet-mediated randomized controlled trial. Trials 2009;10:76.

17. Sandaunet AG. The challenge of fitting in: non-participation and withdrawal from an online self-help group for breast cancer patients. Sociol Health III 2008;30:131-44.

18. Miyamoto $\mathrm{S}$, Henderson $\mathrm{S}$, Young $\mathrm{H}$, et al. Recruiting rural participants for a Telehealth intervention on diabetes self-management. $J$ Rural Health 2013;29:69-77.

19. Kerns JW, Krist AH, Longo DR, et al. How patients want to engage with their personal health record: a qualitative study. BMJ Open 2013;3:e002931.

20. Trief PM, Sandberg J, Izquierdo R, et al. Diabetes management assisted by telemedicine: patient perspectives. Telemed J E Health 2008;14:647-55.

21. Treweek S, Mitchell E, Pitkethly M, et al. Strategies to improve recruitment to randomised controlled trials. Cochrane Database Syst Rev 2010;(4):MR000013.

22. Devlin AM, McGee-Lennon M, O'Donnell CA, et al. Delivering digital health and well-being at scale: lessons learned during the implementation of the dallas Program in the United Kingdom. JAMIA 2016;23:48-59.

23. Lloyd-Williams F, Mair F, Shiels $\mathrm{C}$, et al. Why are patients in clinical trials of heart failure not like those we see in everyday practice? $J$ Clinical Epid 2003;56:1157-62.

24. Mair FS, May C, O'Donnell C, et al. Factors that promote or inhibit the implementation of e-health systems: an explanatory systematic review. Bull World Health Organ 2012;90:357-64.

25. O'Connor S, Mair FS, McGee-Lennon M, et al. Engaging in large-scale digital health technologies and services. What factors hinder recruitment? Stud Health Technol Inform 2015;210:306-10.

26. Damschroder LJ, Aron DC, Keith RE, et al. Fostering implementation of health services research findings into practice: a consolidated framework for advancing implementation science. Implement Sci 2009;4:50.

27. Sheldon TA. Making evidence synthesis more useful for management and policymaking. $J$ Health Serv Res Policy 2005; 10:S1. 
28. Mays N, Pope C, Popay J. Systematically reviewing qualitative and quantitative evidence to inform management and policymaking in the health field. J Health Serv Res Policy 2005;10(Suppl 1):6-20.

29. Finch T, May C, Mair F, et al. Integrating service development with evaluation in telehealthcare: an ethnographic study. $B M J$ 2003;327:1205-8.

30. Dixon-Woods $M$, Booth A Sutton AJ Synthesizing qualitative research: a review of published reports. Qual Res 2007;7:375.

31. Noyes J, Lewin S. Supplemental guidance on selecting a method of qualitative evidence synthesis, and integrating qualitative evidence with Cochrane intervention reviews. In: Noyes J, Booth A, Hannes K, et al., eds. Supplementary Guidance for Inclusion of Qualitative Research in Cochrane Systematic Reviews of Interventions. Cochrane Collaboration Qualitative Methods Group, 2011. www.cochrane-handbook.org (accessed online Aug 2016).

32. Hannes K, Booth A, Harris J, et al. Celebrating methodological challenges and changes: reflecting on the emergence and importance of the role of qualitative evidence in Cochrane reviews. Sys Rev 2013;2:84.

33. Gallacher K, Morrison D, Jani B, et al. Uncovering treatment burden as a key concept for stroke care: a systematic review of qualitative research. PLoS Med 2013;10:e1001473.

34. Morrison D, Wyke S, Agur K, et al. Digital asthma self-management interventions: a systematic review. J Medical Internet Res 2014;16: e51.

35. Carroll C, Booth A, Cooper K. A worked example of "best fit" framework synthesis: a systematic review of views concerning the taking of some potential chemopreventive agents. BMC Med Res Methodol 2011;11:29.

36. Challen $\mathrm{K}$, Lee AC, Booth $\mathrm{A}$, et al. Where is the evidence for emergency planning: a scoping review. BMC Public Health 2012;12:542.

37. May C, Finch T. Implementing, embedding, and integrating practices: an outline of normalization process theory. Sociology 2009;43:535-54.

38. Gallacher K, May CR, Montori VM, et al. Understanding patients' experiences of treatment burden in chronic heart failure using normalization process theory. Ann Fam Med 2011;9:235-43.

39. May CR, Mair F, Finch T, et al. Development of a theory of implementation and integration: normalization process theory. Implement Sci 2009;4:29.

40. McEvoy R, Ballini L, Maltoni S, et al. A qualitative systematic review of studies using the normalization process theory to research implementation processes. Implement Sci 2014;9:2.

41. May CR, Eton DT, Boehmer K, et al. Rethinking the patient: using burden of treatment theory to understand the changing dynamics of illness. BMC Health Serv Res 2014;14:281.

42. Shamseer $\mathrm{L}$, Moher $\mathrm{D}$, Clarke $\mathrm{M}$, et al. Preferred reporting items for systematic review and meta-analysis protocols (PRISMA-P) 2015: elaboration and explanation. BMJ 2015;349:97647.

43. Tong A, Flemming K, Mclnnes E, et al. Enhancing transparency in reporting the synthesis of qualitative research: ENTREQ. BMC Med Res Methodol 2012;12:181.

44. Noyes J, Popay J, Pearson A, et al. Qualitative research and Cochrane reviews. In: Higgins JPT, Green S, eds. Cochrane Handbook for Systematic Reviews of interventions Version 5.1.0. The Cochrane Collaboration, 2011. www.cochrane-handbook.org (accessed online Aug 2016)
45. Ananiadou S, Rea B, Okazaki N, et al. Supporting systematic reviews using text mining. Soc Sci Comp Rev 2009;27:509-23.

46. O'Mara-Eves A, Thomas J, McNaught $\mathrm{J}$, et al. Using text mining for study identification in systematic reviews: a systematic review of current approaches. Syst Rev 2015;4:5.

47. Sandelowski M, Docherty S, Emden C. Focus on qualitative methods Qualitative metasynthesis: issues and techniques. Res Nurs Health 1997;20:365-71.

48. Centre for Reviews and Dissemination (CRD). Incorporating qualitative evidence in or alongside effectiveness reviews. In: Systematic reviews, CRDs guidance for undertaking reviews in healthcare. York, UK: University of York, 2008:219-39.

49. Gallacher K, Jani B, Morrison D, et al. Qualitative systematic reviews of treatment burden in stroke, heart failure and diabetes-Methodological challenges and solutions. BMC Med Res Methodol 2013;13:10.

50. Greenhalgh T, Peacock R. Effectiveness and efficiency of search methods in systematic reviews of complex evidence: audit of primary sources. BMJ 2005;331:1064-5.

51. Johansson T, Wild C. Telerehabilitation in stroke care-a systematic review. J Telemed Telecare 2011;17:1-6.

52. Eysenbach G. The law of attrition. J Medical Internet Res 2005;7:e11.

53. Murray E, White IR, Varagunam M, et al. Attrition revisited: adherence and retention in a web-based alcohol trial. $J$ Medical Internet Res 2013;15:e162.

54. Moher D, Liberati A, Tetzlaff J, et al. Preferred reporting items for systematic reviews and meta-analyses: the PRISMA statement. Int J Surg 2009;151:264-9.

55. Hannes K. Critical appraisal of qualitative research. In: Noyes J, Booth A, Hannes K, et al., eds. Supplementary guidance for inclusion of qualitative research in Cochrane systematic reviews of interventions. Cochrane Collaboration Qualitative Methods Group, 2011. www.cochrane-handbook.org (accessed online Aug 2016).

56. Popay J, Rogers A, Williams G. Rationale and standards for the systematic review of qualitative literature in health services research. Qual Health Res 1998;8:341-51.

57. Dixon-Woods M, Shaw RL, Agarwal S, et al. The problem of appraising qualitative research. Qual Saf Healthcare 2004;13 223-5.

58. Tong A, Sainsbury P, Craig J. Consolidated Criteria for Reporting Qualitative Research (COREQ): a 32-item checklist for interviews and focus groups. Int J Qual Healthcare 2007;19:349-57.

59. Booth A, Hannes K, Harden A, et al. COREQ (Consolidated Criteria for Reporting Qualitative Studies). Guidelines for Reporting Health Research: A User's Manual. 2014:214-26.

60. Kuper $A$, Lingard L, Levinson W. Critically appraising qualitative research. BMJ 2008;337:a1035.

61. Barnett-Page $E$, Thomas J. Methods for the synthesis of qualitative research: a critical review. BMC Med Res Methodol 2009;9:59.

62. Oliver SR, Rees RW, Clarke-Jones L, et al. A multidimensional conceptual framework for analysing public involvement in health services research. Health Expect 2008;11:72-84.

63. Ritchie J, Spencer L. Qualitative data analysis for applied policy research. In: Bryman A, Burgess R, eds. Analysing qualitative data. London: Routledge, 1994:173-94.

64. Mays N, Pope C. Qualitative research: rigour and qualitative research. BMJ 1995;311:109-12.

65. Bazeley P, Jackson K., eds. Qualitative data analysis with NVivo. London: Sage Publications Limited, 2014:1-307. 